\title{
Spin-density matrix elements in hard exclusive muoproduction of $\omega$ mesons at COMPASS
}

\author{
B. Marianski ${ }^{* \dagger}$ \\ for the COMPASS Collaboration \\ National Centre for Nuclear Research Warsaw \\ E-mail: b.marianski@ncbj.gov.pl
}

Spin Density Matrix Elements (SDMEs) have been determined for exclusive $\omega$ meson production on unpolarized protons, in the COMPASS kinematic region of

\begin{abstract}
$1.0(\mathrm{GeV} / \mathrm{c})^{2}<Q^{2}<10.0(\mathrm{GeV} / \mathrm{c})^{2}, 5.0 \mathrm{GeV} / c^{2}<W<17.0 \mathrm{GeV} / c^{2}$ and
$0.01(\mathrm{GeV} / \mathrm{c})^{2}<p_{T}^{2}<0.5(\mathrm{GeV} / \mathrm{c})^{2}$ which corresponds to $\left.\left\langle Q^{2}\right\rangle=2.1(\mathrm{GeV} / \mathrm{c})^{2}\right),\langle W\rangle=$ $7.6 \mathrm{GeV} / c^{2}$ and $\left.\left\langle p_{T}^{2}\right\rangle=0.16(\mathrm{GeV} / \mathrm{c})^{2}\right)$ mean values. Using extracted SDMEs values the contribution of unnatural parity exchange amplitudes and the hypothesis of S-Channel Helicity Conservation (SCHC) were studied. Certain matrix elements e.g. $r_{00}^{5}$ corresponding to transition $\gamma_{T}^{*} \rightarrow V_{L}$ indicate violation of SCHC in exclusive $\omega$ production. A sizable contribution of unnatural parity exchange amplitudes is found for the exclusive $\omega$ meson muoproduction, and there is a clear indication of its decrease with increasing $\mathrm{W}$.
\end{abstract}

23rd International Spin Physics Symposium - SPIN2018 -

10-14 September, 2018

Ferrara, Italy

* Speaker.

${ }^{\dagger}$ Work supported by the NCN Grant 2017/26/M/ST2/00498 


\section{Definition of Spin Density Matrix Elements}

Study of exclusive muoproduction of vector mesons allows investigation of both production mechanism and structure of the nucleon. In particular it gives the possibility to constrain Generalized Parton Distribution (GPDs). At leading twist the chiral-even $G P D s H^{q(g)}(\mathrm{x}, \xi, \mathrm{t})$, $E^{q(g)}(\mathrm{x}, \xi, \mathrm{t}), \tilde{H}^{q(g)}(\mathrm{x}, \xi, \mathrm{t}), \tilde{E}^{q(g)}(\mathrm{x}, \xi, \mathrm{t})$ with helicity of parton unchanged describe exclusive vector meson production on spin 1/2 target. When higher twist effects are included in the distribution amplitude, chiral odd GPDs $H_{T}^{q(g)}(\mathrm{x}, \xi, \mathrm{t}), E_{T}^{q(g)}(\mathrm{x}, \xi, \mathrm{t}), \tilde{H}_{T}^{q(g)}(\mathrm{x}, \xi, \mathrm{t}), \tilde{E}_{T}^{q(g)}(\mathrm{x}, \xi, \mathrm{t})$ with parton helicity flip, appear.

Angular distribution of the decaying particles from vector meson depends on vector meson Spin Density Matrix Elements (SDMEs). These elements on the other hands, are bilinear combinatian of helicity amplitudes $F_{\lambda_{V}} \lambda_{N}^{\prime} \lambda_{\gamma} \lambda_{N}$, where $\lambda_{\gamma}$ is a virtual photon helicity and $\lambda_{V}$ vector meson helicity, while $\lambda_{N}$ and $\lambda_{N}^{\prime}$ are the helicities of the nucleon in the initial and final states, respectively.

In the $\gamma^{*} N$ Centre-of-Mass (CM) system the spin density matrix of the vector meson is given by the von Neumann equation [1] :

$$
\rho_{\lambda_{V} \lambda_{V}^{\prime}}=\frac{1}{2 \mathscr{N}} \sum_{\lambda_{\gamma} \lambda_{\gamma}^{\prime} \lambda_{N} \lambda_{N}^{\prime}} F_{\lambda_{V} \lambda_{N}^{\prime} \lambda_{\gamma} \lambda_{N}} \rho_{\lambda_{\gamma} \lambda_{\gamma}^{\prime}}^{U+L} F_{\lambda_{V}^{\prime} \lambda_{N}^{\prime} \lambda_{\gamma}^{\prime} \lambda_{N},}
$$

where $\mathscr{N}$ is a normalization factor [1-2] and $\rho_{\lambda_{\gamma} \lambda_{\gamma}^{\prime}}^{U+L}$ is the virtual photon spin density matrix, where $\mathrm{U}$ and $\mathrm{L}$ denote unpolarized and longitudinally polarised beam, respectively [2].

After the decomposition of $\rho_{\lambda_{\gamma} \lambda_{\gamma}^{\prime}}^{U+L}$ into the standard set of $3 \times 3$ Hermitian matrices $\Sigma^{\alpha}$, the vector-meson spin density matrix is expressed in terms of a set of nine matrices $\rho_{\lambda_{V} \lambda_{V}^{\prime}}^{\alpha}$ related to various photon polarization states: transversely polarised photon $(\alpha=0, \ldots, 3)$, longitudinally polarised photon $(\alpha=4)$, and terms describing their interference $(\alpha=5, \ldots, 8)$ [1]. In case when contributions of transverse and longitudinal photons cannot be separated, the SDMEs are customarily defined as

$$
\begin{gathered}
r_{\lambda_{V} \lambda_{V}^{\prime}}^{04}=\left(\rho_{\lambda_{V} \lambda_{V}^{\prime}}^{0}+\varepsilon R \rho_{\lambda_{V} \lambda_{V}^{\prime}}^{4}\right)(1+\varepsilon R)^{-1}, \\
r_{\lambda_{V} \lambda_{V}^{\prime}}^{\alpha}=\left\{\begin{array}{l}
\rho_{\lambda_{V} \lambda_{V}^{\prime}}^{\alpha}(1+\varepsilon R)^{-1}, \alpha=1,2,3, \\
\sqrt{R} \rho_{\lambda_{V} \lambda_{V}^{\prime}}^{\alpha}(1+\varepsilon R)^{-1}, \alpha=5,6,7,8 .
\end{array}\right.
\end{gathered}
$$

The quantity $R=d \sigma_{L} / d \sigma_{T}$ is the longitudinal-to-transverse virtual-photon differential cross-section ratio and $\varepsilon$ is the virtual photon polarisation parameter.

The helicity amplitudes are decomposed into the sum of the amplitudes $\mathrm{T}$ for Natural-Parity Exchange (NPE) $\left(\mathrm{P}=(-1)^{J}\right)$ and the amplitudes U for Unnatural-Parity Exchange (UPE) $\left(\mathrm{P}=-(-1)^{J}\right)$, hence given by $F_{\lambda_{V}} \lambda_{N}^{\prime}, \lambda_{\gamma} \lambda_{N}=T_{\lambda_{V}} \lambda_{N}^{\prime}, \lambda_{\gamma} \lambda_{N}+U_{\lambda_{V}} \lambda_{N}^{\prime}, \lambda_{\gamma} \lambda_{N}$. For an unpolarized target there is no interference between NPE and UPE. Also for an unpolarized target there is no linear contribution of nucleon-helicity-flip amplitudes to SDMEs. Bilinear contributions of nucleon helicity flip amplitudes are neglected as they are suppressed by a factor $\left(\sqrt{-t^{\prime}} / M\right)^{2}$, where $t^{\prime}$ is a measure of the transverse momentum of the vector meson with respect to the direction of the virtual photon. So it is convenient to use the following abbreviation $T_{\lambda_{V}} \lambda_{\gamma} \equiv T_{\lambda_{V} \frac{1}{2} \lambda_{\gamma} \frac{1}{2}}$. This reduces the number of amplitudes to nine: the helicity conserving amplitudes $T_{00}, T_{11}, U_{11}$, and the helicity non-conserving amplitudes $T_{01}, T_{10}, T_{1-1}, U_{01}, U_{10}, U_{1-1}$. The dominance of diagonal transitions is called S-Channel Helicity Conservation (SCHC). 
For a longitudinally polarized beam and unpolarized target there are 23 SDMEs; 15 which do not depend on beam polarization, and 8 which depend on beam polarization. They can be determined from a fit of the angular distribution of produced $\omega$ and pions from its decay.

The values of the SDMEs can be used to establish the hierarchy of the helicity amplitudes that are used to describe exclusive $\omega$ production. They are also used to test the SCHC hypothesis and to investigate the contribution of the unnatural parity exchange mechanism in $\omega$ production. Also they allow to test different GPD models.

\section{Experiment and data processing}

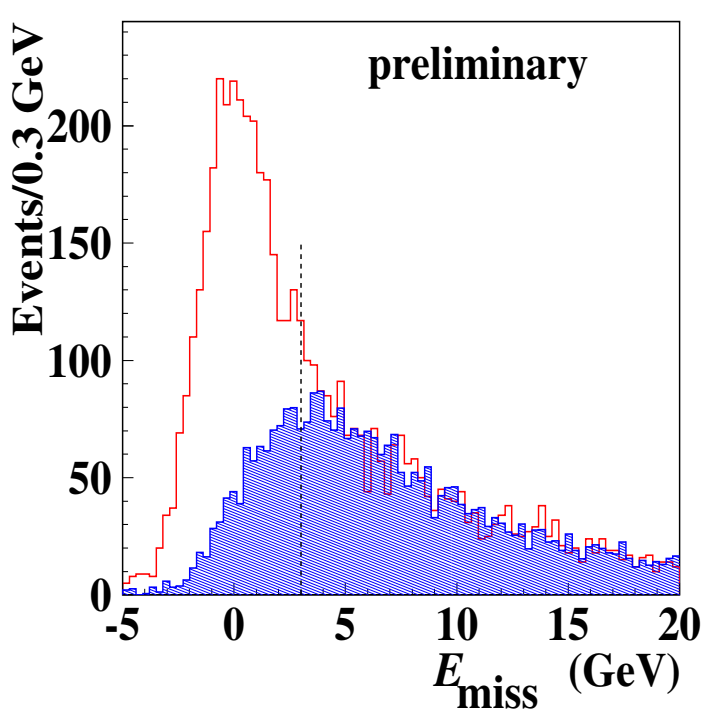

Figure 1: The $E_{\text {miss }}$ distribution of data for exclusive $\omega$ production (red line) is compared with the SIDIS $E_{\text {miss }}$ distribution from LEPTO MC (shaded blue area).

The analysis is based on data taken by COMPASS in 2012 with the $160 \mathrm{GeV}$ muon polarized beam and the unpolarized liquid hydrogen target. The studied process of exclusive $\omega$ meson production is the following:

$$
\begin{aligned}
& \mu N \rightarrow \mu^{\prime} N^{\prime} \omega \\
& \begin{array}{rlrl}
\longrightarrow & \pi^{+} \pi^{-} \pi^{0} & \mathrm{BR} \approx 89 \% \\
\llcorner & & \mathrm{BR} \approx 99 \%
\end{array}
\end{aligned}
$$

So an event accepted for further analysis should consist of a track of scattered muon two tracks of hadrons with opposite charges and two neutral clusters.

The exclusivity of the omega production is characterized by missing energy $E_{\text {miss }}=\left(M_{X}^{2}-M_{p}^{2}\right) / 2 M_{p}$, with the missing mass squared $M_{X}^{2}=\left(p+q-p_{\pi^{+}}-p_{\pi^{-}}-p_{\pi^{0}}\right)^{2}$, where $p, q, p_{\pi^{+}}, p_{\pi^{-}}$and $p_{\pi^{0}}$ are the four-momenta of the proton, virtual photon and each of the three $\omega$-decay pions, respectively.

For the exclusive reaction the target nucleon remains intact, which corresponds to $E_{\text {miss }}=0.0 \mathrm{GeV}$. Taking into account the spectrometer resolution the missing energy is required 
to be in the interval $-3.0 \mathrm{GeV}<E_{\text {miss }}<3.0 \mathrm{GeV}$. In Fig. 1 the missing energy distribution is shown by the red histogram. Blue dashed area coresponds to $E_{\text {miss }}$ distribution for the LEPTO MC simulation reweighted in the same way as in Ref. [3]. The fraction of background for the entire kinematic region is $20 \%$. In Fig. 2 invariant mass of $\gamma \gamma$ and distributions of $\pi^{+} \pi^{-} \pi^{0}$ invariant mass are shown. After all cuts we have 3060 exclusive events.
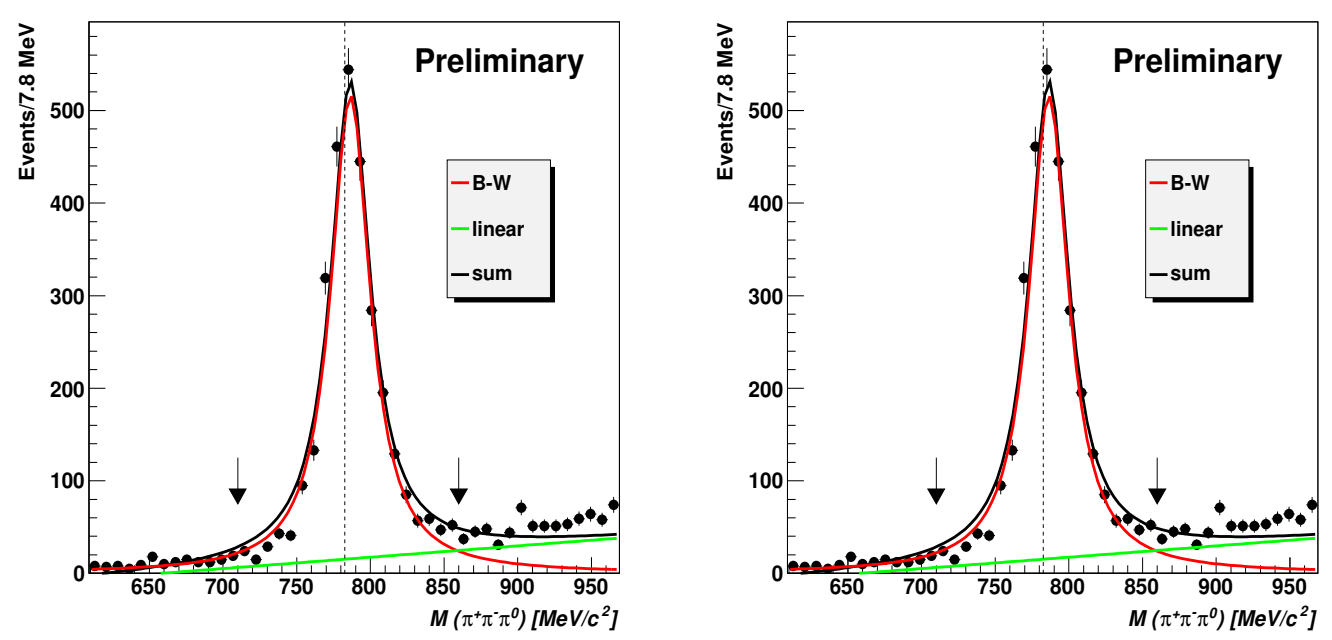

Figure 2: Left panel: Distributions of $\gamma \gamma$ invariant mass (left) fitted by Gaussian function and linear background. Dashed vertical line denotes PDG value. Right panel: Distributions of $\pi^{+} \pi^{-} \pi^{0}$ invariant mass (right) fitted by Breit-Wigner function and linear background. Vertical arrows denote cuts for the invariant mass. Dashed vertical line denotes PDG value.

\section{Test of the SCHC Hypothesis}

The SDMEs for the entire kinematic region have been determined in the COMPASS kinematic region of $1.0(\mathrm{GeV} / \mathrm{c})^{2}<Q^{2}<10.0(\mathrm{GeV} / \mathrm{c})^{2}, 5.0 \mathrm{GeV} / c^{2}<W 17.0 \mathrm{GeV} / c^{2}$ and $0.01(\mathrm{GeV} / \mathrm{c})^{2}<p_{T}^{2}<0.5(\mathrm{GeV} / \mathrm{c})^{2}$, which corresponds to $\left\langle Q^{2}\right\rangle=2.1(\mathrm{GeV} / \mathrm{c})^{2},\langle W\rangle=7.6 \mathrm{GeV} / c^{2}$ and $\left\langle p_{T}^{2}\right\rangle=0.16(\mathrm{GeV} / \mathrm{c})^{2}$. Here, $Q^{2}$ represents the negative-square of the virtual-photon fourmomentum, $W$ the invariant mass of the photon-nucleon system and $p_{T}^{2}$ the squared transverse momentum of $\omega$ with respect to virtual photon $\gamma^{*}$. The SDMEs of the $\omega$ meson for the integrated data are presented in Fig. 3. The presented SDMEs are divided into five classes corresponding to different helicity transitions. Class A corresponds to the transition of longitudinal virtual photons to longitudinal mesons $\gamma_{L}^{*} \rightarrow V_{L}$ and transverse virtual photons to transverse mesons $\gamma_{T}^{*} \rightarrow V_{T}$. Class B corresponds to the interference of these two transitions. Class $C$ corresponds to the $\gamma_{T}^{*} \rightarrow V_{L}$ transition, class $\mathrm{D}$ to the $\gamma_{L}^{*} \rightarrow V_{T}$ transition, and class $\mathrm{E}$ to the $\gamma_{T}^{*} \rightarrow V_{-T}$ transition.

In the case of SCHC only the seven SDMEs of class A and class B $\left(r_{00}^{04}, r_{1-1}^{1}, \operatorname{Im}\left\{r_{1-1}^{2}\right\}\right.$, $\left.\operatorname{Re}\left\{r_{10}^{5}\right\}, \operatorname{Im}\left\{r_{10}^{6}\right\}, \operatorname{Im}\left\{r_{10}^{7}\right\}, \operatorname{Re}\left\{r_{10}^{8}\right\}\right)$, are not restricted to be zero, but six of them have to obey the following relations [1]: $r_{1-1}^{1}=-\operatorname{Im}\left\{r_{1-1}^{2}\right\}, \operatorname{Re}\left\{r_{10}^{5}\right\}=-\operatorname{Im}\left\{r_{10}^{6}\right\}, \operatorname{Im}\left\{r_{10}^{7}\right\}=\operatorname{Re}\left\{r_{10}^{8}\right\}$.

For these SDMEs we found: $r_{1-1}^{1}+\operatorname{Im}\left\{r_{1-1}^{2}\right\}=-0.01 \pm 0.038 \pm 0.047$, $\operatorname{Re}\left\{r_{10}^{5}\right\}+\operatorname{Im}\left\{r_{10}^{6}\right\}=0.044 \pm 0.011 \pm 0.013, \operatorname{Im}\left\{r_{10}^{7}\right\}-\operatorname{Re}\left\{r_{10}^{8}\right\}=-0.088 \pm 0.110 \pm 0.196$. 


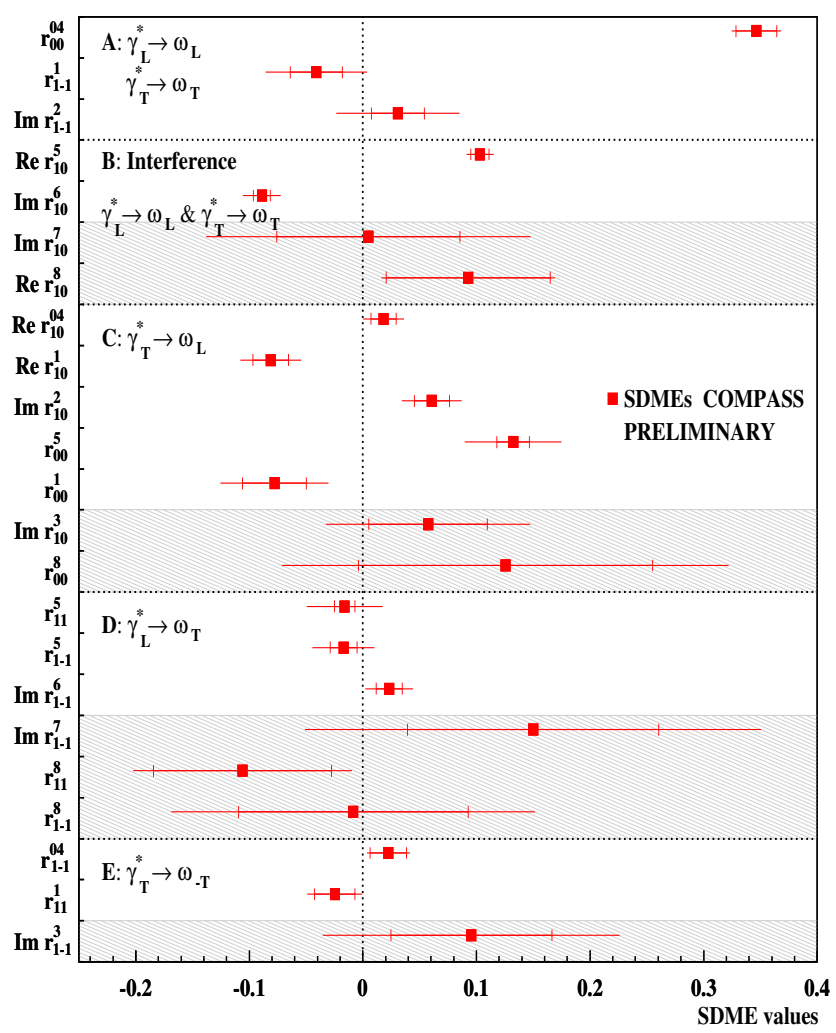

Figure 3: The 23 SDMEs extracted for exclusive $\omega$ production in the entire COMPASS kinematic region with $\left\langle Q^{2}\right\rangle=2.1(\mathrm{GeV} / \mathrm{c})^{2},\langle W\rangle=7.6 \mathrm{GeV} / \mathrm{c}^{2}$ and $\left\langle p_{T}^{2}\right\rangle=0.16(\mathrm{GeV} / \mathrm{c})^{2}$ mean values. The inner error bars represent the statistical uncertainties, while the outer ones indicate the statistical and systematic uncertainties added in quadrature. SDMEs measured with unpolarized (polarized) beam are displayed in the unshaded (shaded) areas.

These relations for classes A and B fulfill the requirements of SCHC. All other SDMEs are required by SCHC to be zero. However, from Fig. 3 one can see that few SDMEs of class C, like $\operatorname{Re} r_{00}^{5}, \operatorname{Re} r_{10}^{1}, \operatorname{Im} r_{10}^{2}$, are inconsistent with the hypothesis of SCHC.

\section{Dependence of selected SDMEs on W}

Beside of SDMES for the entire kinematic region, the kinematic dependences of SDMEs on $\mathrm{Q}^{2}, \mathrm{p}_{T}^{2}$ and $\mathrm{W}$ were also determined. In Table 1 values of SDMEs $r_{1-1}^{1}$ and $\operatorname{Im} r_{1-1}^{2}$ as a function of $\langle W\rangle$ are shown. The difference between these two SDMEs expressed by helicity amplitudes is the following: 


\begin{tabular}{|c|c|c|c|}
\hline$\langle W\rangle\left(\mathrm{GeV} / \mathrm{c}^{2}\right)$ & 5.9 & 7.1 & 9.9 \\
\hline$r_{1-1}^{1}$ & $-0.134 \pm 0.043 \pm 0.32$ & $-0.044 \pm 0.036 \pm 0.33$ & $0.052 \pm 0.038 \pm 0.047$ \\
\hline $\mathrm{Im} r_{1-1}^{2}$ & $0.139 \pm 0.044 \pm 0.46$ & $0.037 \pm 0.036 \pm 0.24$ & $-0.098 \pm 0.038 \pm 0.033$ \\
\hline $\mathrm{u}_{1}$ & $1.05 \pm 0.14 \pm 0.039$ & $0.90 \pm 0.12 \pm 0.021$ & $0.60 \pm 0.11 \pm 0.12$ \\
\hline
\end{tabular}

Table 1: Dependence of SDMEs $r_{1-1}^{1}, \operatorname{Im} r_{1-1}^{2}$ and $\mathrm{u}_{1}$ on $W$

$$
\operatorname{Im}\left\{r_{1-1}^{2}\right\}-r_{1-1}^{1}=\frac{1}{\mathscr{N}}\left(-\left|T_{1 \frac{1}{2} 1 \frac{1}{2}}\right|^{2}-\left|T_{1-\frac{1}{2} 1 \frac{1}{2}}\right|^{2}+\left|U_{1 \frac{1}{2} 1 \frac{1}{2}}\right|^{2}+\left|U_{1-\frac{1}{2} 1 \frac{1}{2}}\right|^{2}\right) .
$$

Where $\mathscr{N}$ is a normalization factor [4]. As mentioned in the first section $T_{1-\frac{1}{2} 1 \frac{1}{2}}, U_{1-\frac{1}{2} 1 \frac{1}{2}}$, amplitudes corresponding to nucleon helicity spin flip are suppresed and can be omitted. Using the abbreviation introduced in the first section we get :

$$
\operatorname{Im}\left\{r_{1-1}^{2}\right\}-r_{1-1}^{1}=\frac{1}{\mathscr{N}}\left(-\left|T_{1 \frac{1}{2} 1 \frac{1}{2}}\right|^{2}+\left|U_{1 \frac{1}{2} 1 \frac{1}{2}}\right|^{2}\right)=\frac{1}{\mathscr{N}}\left(-\left|T_{11}\right|^{2}+\left|U_{11}\right|^{2}\right) .
$$

From Tab. 1 one can see that for $\langle W\rangle=5.9 \mathrm{GeV} / \mathrm{c}^{2},\left\langle p_{T}^{2}\right\rangle=0.16(\mathrm{GeV} / \mathrm{c})^{2}$ the difference of the SDMEs is large and positive $\left(\operatorname{Im} r_{1-1}^{2}-r_{1-1}^{1}>0 \Rightarrow U_{11}>T_{11}\right)$ so the unnatural parity amplitude is greater than natural parity one.

For $\langle W\rangle=7.1 \mathrm{GeV} / \mathrm{c}^{2},\left\langle p_{T}^{2}\right\rangle=0.16(\mathrm{GeV} / \mathrm{c})^{2}$ the values of SDMEs are close to zero and their difference is also close to zero $\left(\operatorname{Im} r_{1-1}^{2}-r_{1-1}^{1} \approx 0 \Rightarrow U_{11} \approx T_{11}\right)$ and the NPE amplitude is almost equal to the UPE amplitude.

For $\langle W\rangle=9.9 \mathrm{GeV} / \mathrm{c}^{2},\left\langle p_{T}^{2}\right\rangle=0.16(\mathrm{GeV} / \mathrm{c})^{2}$ one observe that the SDMEs reverse the signs, which indicate that $r_{1-1}^{2}-r_{1-1}^{1} \leq 0 \Rightarrow U_{11} \leq T_{11}$. The UPE processes become smaller than NPE ones. It is consistent with expectation that the fractional contribution of UPE (e.g. pion exchange) decreases as $\mathrm{W}$ increases.

This is also confirmed by the $W$ dependence of the parameter $u_{1}$ which is a measure of UPE contribution, defined as $u_{1}=1-r_{00}^{04}+2 r_{1-1}^{04}-2 r_{11}^{1}-2 r_{1-1}^{1}$. It can be expressed by the helicity amplitudes $u_{1}=\widetilde{\Sigma} \frac{4 \varepsilon\left|U_{10}\right|^{2}+2\left|U_{11}+U_{-11}\right|^{2}}{\mathscr{N}}$. The numerator of $u_{1}$ depends only on UPE amplitudes, thus $u_{1}>0$ is a signal of UPE contribution. We see from the fourth row of Tab. 1 that the UPE contribution decreases with increasing $W$ but it is still large as $\left|U_{11}\right|$ is comparable with $\left|T_{11}\right|$.

\section{References}

[1] K. Schilling and G. Wolf, Nucl. Phys. C 61 (361) 1973.

[2] A. Airapetian et al. (HERMES Collaboration), Eur. Phys. J. C 62 (659) 2009.

[3] COMPASS Collaboration, Nucl. Phys. B 915 (454) 2017.

[4] A. Airapetian et al. (HERMES Collaboration), Eur. Phys. J. C 74 (3110) 2014; Erratum: Eur. Phys. J. C 76, 162 (2016). 